\title{
Application of the Stationary Wavelet Transform to Reduce Power-line Interference in Atrial Electrograms
}

\author{
Miguel Martínez-Iniesta ${ }^{1}$, Juan Ródenas $^{1}$, Raúl Alcaraz $^{1}$, José J. Rieta ${ }^{2}$ \\ ${ }^{1}$ Research Group in Electronic, Biomedical and Telecomm. Eng., Univ. of Castilla-La Mancha, Spain \\ ${ }^{2}$ BioMIT.org, Electronic Engineering Department, Universitat Politecnica de Valencia, Spain
}

\begin{abstract}
The atrial electrical activity analysis is nowadays playing a key role to improve current knowledge about the mechanisms triggering and maintaining atrial arrhythmias, such as atrial fibrillation (AF). Indeed, the intraatrial electrograms (EGMs) provide essential information to guide the prevailing cornerstone treatment of $A F$, i.e., catheter ablation. Bearing in mind that cardiac electrophysiology laboratories are plenty of technology, such a kind of operating rooms are a very adverse environment when it comes to avoid power-line interference in EGM recordings. However, the reduction of this nuisance signal deserves more attention. Thus, aimed at reducing powerline noise but, at the same time, preserving the original EGM morphology, a novel algorithm based on the stationary Wavelet transform (SWT) is proposed. To validate the method, 150 bipolar EGMs with 10 seconds in length have been synthesized with different noise levels. The original and denoised EGMs have then been compared by means of an adaptive signed correlation index (ASCI), computed both in time and frequency domains. The obtained results have shown improvements between $9 \%$ and $17 \%$ for the proposed method regarding a reference notch filtering. Consequently, this new algorithm may enable more reliable and truthful further analyses of atrial EGMs.
\end{abstract}

\section{Introduction}

The extracellular electrogram (EGM), recorded directly from the heart, is the hallmark of invasive cardiac electrophysiology and provides information about the electric status of the underlying myocardium [1]. This signal is generated by depolarization of cardiomyocytes that provokes transmembrane currents in extracellular space and potential differences due to electric resistance of the extracellular medium [1]. In contrast to the healthy myocardium where the shape of the EGM is simple, under pathological conditions this recording consists of multiple components and long duration, which have been attributed to abnormal conduction and arrhythmogenicity [2]. Hence, the EGM analysis plays a key role in the improvement of current knowledge about the mechanisms triggering and maintaining atrial arrhythmias, such as atrial fibrillation (AF) [3].

Indeed, although AF is the most common arrhythmia in clinical practice and affects approximately $2 \%$ of general adult population, its pathophysiological mechanisms are not fully known $[4,5]$. This aspect makes its diagnostic and treatment highly complex and poorly effective [4] and, therefore, more efforts are required to improve the management of AF [6]. To this respect, the first-line therapy for this disease, i.e., catheter ablation, still provides notably low long-term success rates and many experts claim for the need of more advanced processing and interpretation of the EGMs acquired during the procedure to determine more precisely the atrial areas to be targeted [7].

However, the EGM recording is often characterized by a low amplitude and tainted by notable nuisance interferences, since it is acquired in the presence of numerous sources of electric noise [8]. Hence, to develop further accurate and trustful analyses, signal preprocessing is an essential step [9]. Within this context, the power-line interference should receive special attention, because it is located within the EGM bandwidth and, thus, its isolated elimination is difficult to perform [10]. Despite this, comercial recording systems often incorpore low-order simple notch filters, which remove important local cardiac components along with the nuisance signal [9]. In the present work, an algorithm based on the stationary Wavelet transform (SWT) is proposed to reduce power-line interference and, at the same time, preserve the original EGM morphology as much as possible.

\section{Methods}

\subsection{Study population}

The proposed algorithm was validated making use of a set of synthesized bipolar EGMs. The use of these signals allows to quantify accurately the achieved noise reduction and the waveform integrity preservation. Thus, an 

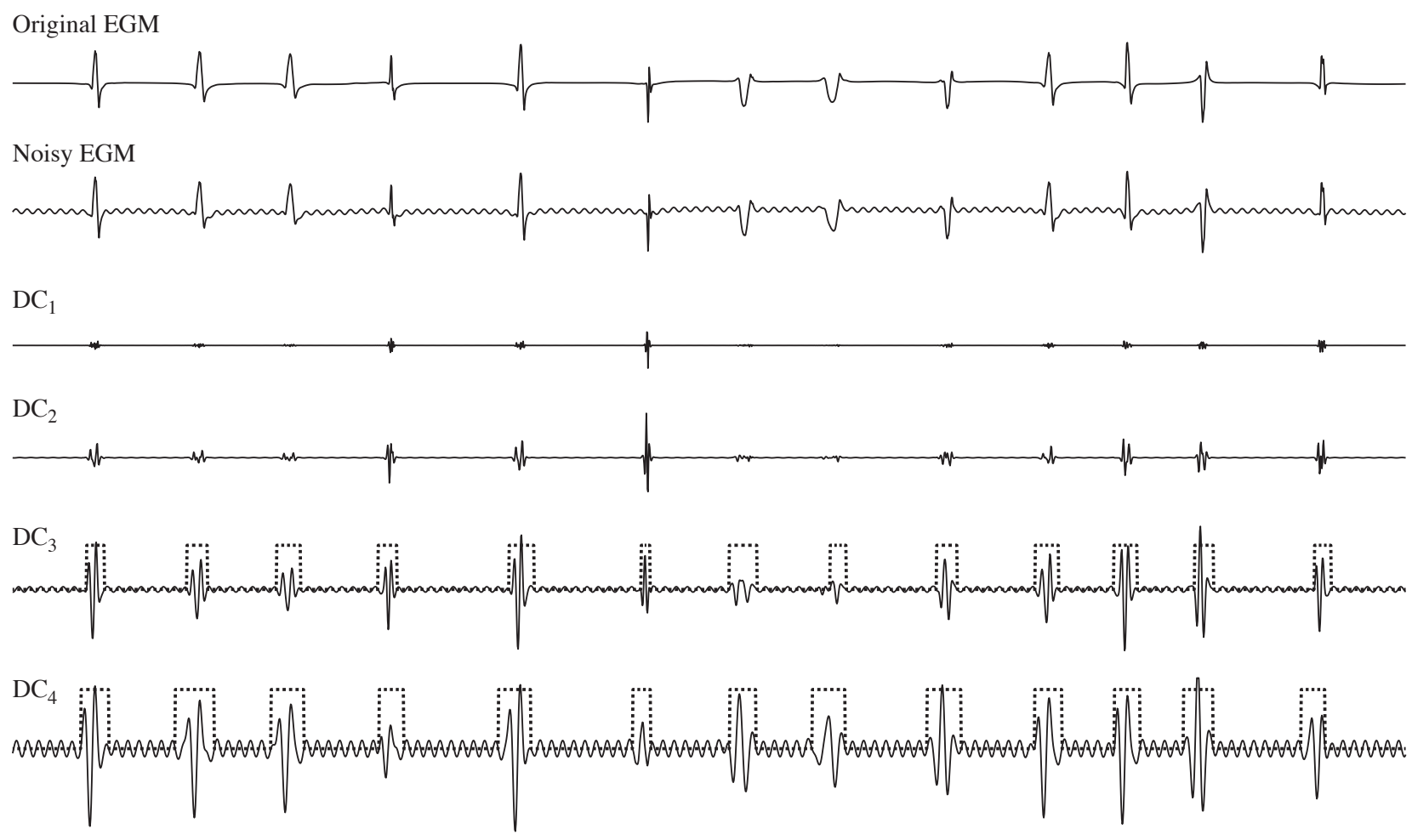

Figure 1. Graphical summary about how the proposed SWT-based algorithm works. To remove the power-line interference, atrial activations are detected and windowed in scales $3\left(\mathrm{DC}_{3}\right)$ and $4\left(\mathrm{DC}_{4}\right)$.

approach similar to the presented by Oesterlin et al. [11] was used to synthesize 150 EGM recordings with a duration of 10 seconds. Next, to obtain recordings with signalto-interference ratios (SIR) of $25,20,15,10$ and $5 \mathrm{~dB}$, a signal centered on $50 \mathrm{~Hz}$ and containing two harmonics was generated and added to the clean synthesized EGM recordings. Next, random variations in amplitude and frequency were performed within very limited ranges for the two added harmonics of $50 \mathrm{~Hz}$.

\subsection{SWT-based denoising algorithm}

Wavelet transform (WT) has been proven to be an useful tool for the denoising of many physiological signals, since it is able to deal successfully with transients, aperiodicities and other non-stationary components [12]. Briefly, this tool decomposes a signal into different scales emphasizing its main time and frequency features [12]. An interesting variant of classical WT is its stationary version, i.e. SWT, which overcomes the lack of translation-invariance [13]. More precisely, each scale or detail coefficient (DC) is only obtained through a bank of low- and high-pass finite impulse response filters, without decimation stages. Thus, every DC presents the same length as the original signal.

Making use of this tool, the EGM was decomposed into four levels with a second-order Coiflet function as wavelet mother. Given that the power-line interference was mainly concentrated on scales 3 and 4 (because they cover a frequency range between 31 and $62 \mathrm{~Hz}$, approximately), atrial activations in these DCs were detected by thresholding and windowed to preserve their morphology, such as the example in Fig. 1 shows. Thus, the noise out of the established windows was removed and the denoised signal was finally reconstructed from the resulting wavelet coefficients.

\subsection{Performance assessment}

As a reference for comparison, a similar notch filter to the one incorporated in many commercial recording systems has been replicated by using a third-order Butterworth structure. It was designed with a $2 \mathrm{~Hz}$ bandwidth centered on the frequency of $50 \mathrm{~Hz}$ and applied to the EGM signal in a forward/backward fashion.

To quantify noise reduction and morphology preservation in the resulting EGM signal for the two analyzed algorithms, an adaptive signed correlation index (ASCI) was computed both in time and frequency domains. This index was used instead of the common Pearson's correlation coefficient, because additionally it takes into consideration the amplitude differences between the two compared 
Table 1. Average values of ASCI obtained for the proposed SWT-based denosing algorithm and the reference notch filtering both from time and frequency domains.

\begin{tabular}{llccccc}
\hline \hline \multirow{2}{*}{ Method } & & \multicolumn{5}{c}{ SIR (dB) } \\
\cline { 3 - 7 } & ASCI (\%) & $\mathbf{2 5}$ & $\mathbf{2 0}$ & $\mathbf{1 5}$ & $\mathbf{1 0}$ & $\mathbf{5}$ \\
\hline \multirow{2}{*}{ SWT-based denoising } & Time & $90.3 \pm 4.3$ & $89.8 \pm 4.5$ & $88.2 \pm 4.9$ & $85.5 \pm 5.1$ & $82.3 \pm 5.7$ \\
& Frequency & $98.2 \pm 1.3$ & $98.1 \pm 1.4$ & $98.0 \pm 1.3$ & $97.6 \pm 1.4$ & $96.6 \pm 1.6$ \\
\hline \multirow{2}{*}{ Notch filtering } & Time & $73.3 \pm 7.5$ & $73.3 \pm 7.5$ & $73.3 \pm 7.6$ & $73.3 \pm 7.6$ & $73.2 \pm 7.6$ \\
& Frequency & $95.4 \pm 0.9$ & $95.4 \pm 0.9$ & $95.4 \pm 1.0$ & $95.4 \pm 0.9$ & $95.5 \pm 0.9$ \\
\hline \hline
\end{tabular}

signals. From a mathematical point of view, if the original clean EGM is named $x(n)$ and the denoised recording $\hat{x}(n)$, the ASCI is computed as

$$
\operatorname{ASCI}[x(n), \hat{x}(n)]=\frac{1}{L} \sum_{k=1}^{L} x(k) \otimes \hat{x}(k),
$$

where $L$ is the number of samples both for $x(n)$ and $\hat{x}(n)$ and the operator $\otimes$ is defined as

$$
x(n) \otimes \hat{x}(n)=\left\{\begin{aligned}
1 & \text { if }|x(n)-\hat{x}(n)| \leq \xi, \\
-1 & \text { if }|x(n)-\hat{x}(n)|>\xi .
\end{aligned}\right.
$$

The threshold $\xi$ was experimentally set to $5 \%$ of the standard deviation of $x(n)$.

\section{Results}

Table 1 summarizes the average values of ASCI obtained for the proposed SWT-based denoising algorithm and the reference notch filtering both from time and frequency domains. As can be seen, whereas the notch filtering provided lower and almost fixed values of ASCI regardless of the noise level, the SWT-based method reported slightly altered time and frequency waveforms as the SIR decreased. Nonetheless, the proposed algorithm always provided notably cleaner and more unaltered resulting EGMs than the reference filtering. As a graphical example, Fig. 2 displays the resulting signals for both denoising methods in time and frequency domains, when a SIR of $15 \mathrm{~dB}$ was considered.

\section{Discussion and conclusions}

The EGM preprocessing plays a key role to reach further accurate and trustful analyses. However, this aspect has not received broad attention in the literature. In fact, the simple notch filter included by commercial recording systems is widely used to reduce power-line interference in the EGM recording, although it modifies notably the signal morphology both in time and frequency domains, such as the presented results have proven. Moreover, it is worth noting that this filtering provokes a similar alteration in the EGM recording regardless of the noise level, thus completely disturbing even those signals with a very limited interference. In this way, clinically useful information could be lost and, additionally, the connection between the EGM morphology and the underlying electrophysiological mechanisms of AF could be blurred.

On the contrary, the proposed SWT-based algorithm has provided a promising ability to reduce the power-line interference, even for very low values of SIR of 5 and $10 \mathrm{~dB}$. Moreover, as Fig. 2 shows, the EGM morphology both in time and frequency domains have been mainly preserved, thus enabling further realistic and reliable electrophysiological studies. Another interesting feature of this method is its simplicity, so that it may be easily incorporated to commercial recording systems. However, the algorithm requires the detection of atrial activations in scales 3 and 4 , which is not always simple for complex and fragmented EGMs [14]. Consequently, additional experiments focused on these kinds of recordings will be developed in future.

\section{Acknowledgements}

Research supported by grants TEC2014-52250-R and DPI2017-83952-C3 MINECO/AEI/FEDER, UE, and PEII-2014-028-P from Junta de Comunidades de CastillaLa Mancha.

\section{References}

[1] de Bakker JMT, Wittkampf FHM. The pathophysiologic basis of fractionated and complex electrograms and the impact of recording techniques on their detection and interpretation. Circ Arrhythm Electrophysiol Apr 2010;3(2):20413.

[2] Issa ZF, Miller JW, Zipes DP. Clinical arrhythmology and electrophysiology: a comparison to Braunwald's heart disease. (Chapter 4). 2nd edition. Elsevier, 2012.

[3] Heijman J, Algalarrondo V, Voigt N, Melka J, Wehrens XHT, Dobrev D, Nattel S. The value of basic research insights into atrial fibrillation mechanisms as a guide to therapeutic innovation: a critical analysis. Cardiovasc Res Apr 2016;109(4):467-79.

[4] Schnabel RB, Yin X, Gona P, Larson MG, Beiser AS, McManus DD, Newton-Cheh C, Lubitz SA, Magnani JW, Elli- 


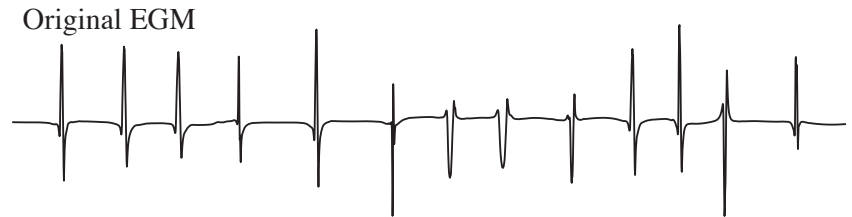

Noisy EGM

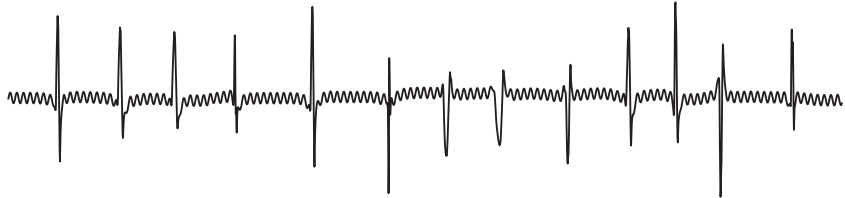

SWT-based denoised EGM

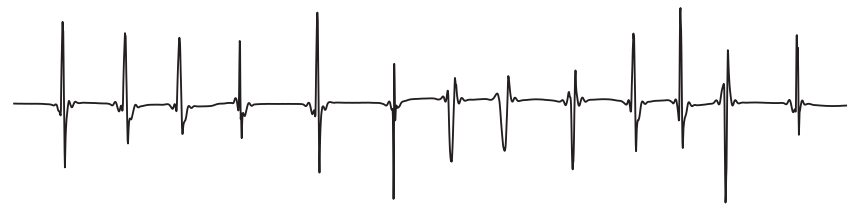

Notch filtered EGM

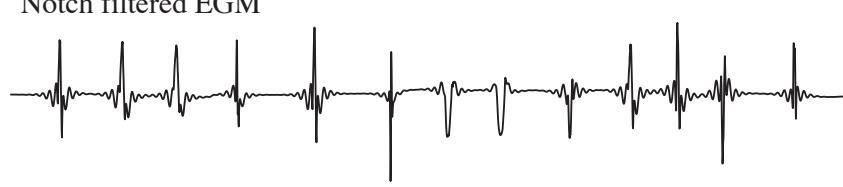

(a)
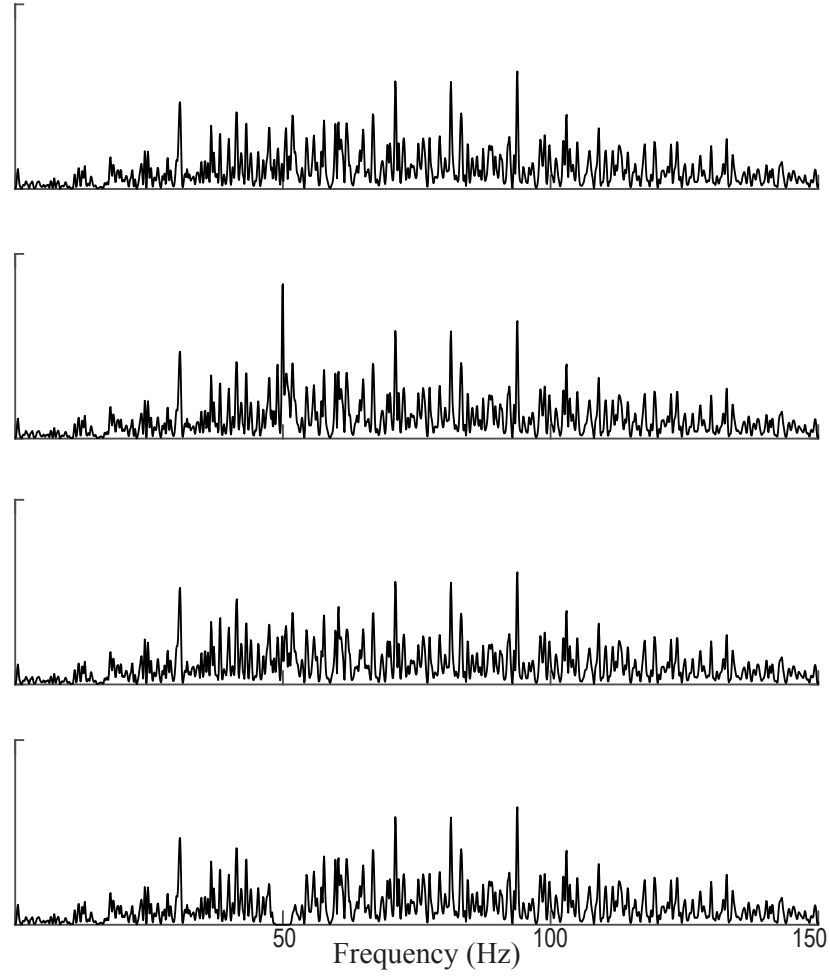

(b)

Figure 2. Typical example of the resulting EGM signals for the two analyzed denoising methods both in time (a) and frequency (b) domains, when a SIR of $15 \mathrm{~dB}$ was considered.

nor PT, Seshadri S, Wolf PA, Vasan RS, Benjamin EJ, Levy D. 50 year trends in atrial fibrillation prevalence, incidence, risk factors, and mortality in the Framingham Heart Study: a cohort study. Lancet Jul 2015;386(9989):154-62.

[5] Schotten U, Dobrev D, Platonov PG, Kottkamp H, Hindricks G. Current controversies in determining the main mechanisms of atrial fibrillation. J Intern Med May 2016; 279(5):428-38.

[6] Van Wagoner DR, Piccini JP, Albert CM, Anderson ME, Benjamin EJ, et al. Progress toward the prevention and treatment of atrial fibrillation: A summary of the Heart Rhythm Society Research Forum on the Treatment and Prevention of Atrial Fibrillation, Washington, DC, December 9-10, 2013. Heart Rhythm Jan 2015;12(1):e5-e29.

[7] Latchamsetty R, Morady F. Atrial fibrillation ablation. Annu Rev Med Aug 2017;

[8] Venkatachalam KL, Herbrandson JE, Asirvatham SJ. Signals and signal processing for the electrophysiologist. Part I: electrogram acquisition. Circ Arrhythm Electrophysiol Dec 2011;4(6):965-73.

[9] Venkatachalam KL, Herbrandson JE, Asirvatham SJ. Signals and signal processing for the electrophysiologist. Part II: signal processing and artifact. Circ Arrhythm Electrophysiol Dec 2011;4(6):974-81.

[10] Jadidi A, Lehrmann H, Weber R, Park C, Arentz T. Optimising signal acqusistion and recording in an electrophysi- ology laboratory. Cardiac Electrophysiology Clinics 2013; 5:137-142.

[11] Oesterlein TG, Lenis G, Rudolph DT, Luik A, Verma B, Schmitt C, Dössel O. Removing ventricular far-field signals in intracardiac electrograms during stable atrial tachycardia using the periodic component analysis. J Electrocardiol 2015;48(2):171-80.

[12] Addison PS. Wavelet transforms and the ECG: a review. Physiol Meas Oct 2005;26(5):R155-99.

[13] Lieb F, Stark HG, Thielemann C. A stationary wavelet transform and a time-frequency based spike detection algorithm for extracellular recorded data. J Neural Eng Jun 2017;14(3):036013.

[14] Ng J, Sehgal V, Ng JK, Gordon D, Goldberger JJ. Iterative method to detect atrial activations and measure cycle length from electrograms during atrial fibrillation. IEEE Trans Biomed Eng Feb 2014;61(2):273-8.

Address for correspondence:

Miguel Martínez Iniesta

E.I. Industriales, Campus Universitario, 02071, Albacete, Spain

Phone: +34-967-599-200 Ext. 2555

Fax: +34-967-599-224

e-mail: miguel.martinez@uclm.es 